This document is the Accepted Manuscript version of a Published Work that appeared in final form in

ACS sensors , copyright (C 2020 American Chemical Society after peer review and technical editing by $\mathrm{t}$ he publisher. To access the final edited and published work see

https://pubs.acs.org/doi/10.1021/acssensors.0c00418

\title{
Selective ultrasensitive optical fiber nanosensors based on plasmon resonance energy transfer
}

Javier Barroso ${ }^{1,2 \#}$, Angel Ortega-Gomez ${ }^{3 \#}$, Alba Calatayud-Sanchez ${ }^{1,2}$, Joseba Zubia ${ }^{3}$, Fernando Benito-Lopez ${ }^{2}$, Joel Villatoro*3,4, Lourdes Basabe-Desmonts*1,4

${ }^{1}$ BIOMICs-microfluidics Research Group, Microfluidics Cluster UPV/EHU, University of the Basque Country UPV/EHU, Vitoria-Gasteiz, Alava, Spain, 01006.

${ }^{2}$ AMMa LOAC Research Group, Microfluidics Cluster UPV/EHU, University of the Basque Country UPV/EHU, Vitoria-Gasteiz, Alava, Spain, 01006.

${ }^{3}$ Department of Communications Engineering, University of the Basque Country UPV/EHU, 48013, Bilbao, Spain.

${ }^{4}$ IKERBASQUE, Basque Foundation for Science, Bilbao, Spain, 48013.

\# Both authors contributed equally to this work.

${ }^{*}$ Corresponding authors, e-mail addresses: lourdes.basabe@ehu.eus and joel.villatoro@ehu.es 


\section{ABSTRACT}

The facet of optical fibers coated with nanostructures enable the development of ultraminiature and sensitive (bio)chemical sensors. The reported sensors until now lack of specificity and the fabrication methods offer poor reproducibility. Here, we demonstrate that by transforming the facet of conventional multimode optical fibers onto plasmon resonance energy transfer (PRET) antenna surfaces the specificity issues may be overcome. To do so, a low cost chemical approach was developed to immobilize gold nanoparticles on the optical fiber facet in a reproducible and controlled manner. Our nanosensors are highly selective as PRET is a nanospectroscopic effect that only occurs when the resonant wavelength of the nanoparticles matches that of the target parameter. As an example, we demonstrate the selective detection of picomolar concentrations of copper ions in water. Our sensor is 1,000 times more sensitive than state of the art technologies. An additional advantage of our nanosensors is their simple interrogation; it comprises of a lowpower light emitting diode, a multimode optical fiber coupler, and a miniature spectrometer. We believe that the PRET-based fiber optic platform reported here may pave the way of the development of a new generation of ultra-miniature, portable, and hypersensitive and selective (bio)chemical sensors.

Keywords: optical fiber, plasmonics, chemosensor, nanoparticles, metal ion 
The localized surface plasmon resonance (LSPR) phenomenon constitutes the basis of highly sensitive optical (bio)chemical sensors. So far, a myriad of LSPR-based sensors have been reported in the literature ${ }^{1-4}$. The operating principle of such nanosensors is based on the detection of the LSPR spectral position upon chemical or biomolecular interactions that occur on the surface of the plasmonic nanostructure.

Among the multiple platforms and alternatives to devise an LSPR sensor, the flat end of a conventional optical fiber coated with gold nanoparticles (AuNPs) or other plasmonic nanostructures present the highest degree of miniaturization. Such optical fiber nanoprobes have important advantages for optical sensing. Their miniature dimensions make them ideal for applications where the amount of sample is extremely small while optical fibers confer such sensors flexibility and capability for remote or in situ sensing ${ }^{5}$.

Nanopatterning the microscopic facet of an optical fiber by means of conventional nanofabrication techniques is challenging. So far, different fabrication processes based on electron beam lithography $y^{6-8}$ and focus ion beam ${ }^{9-11}$ have been used to make plasmonic nanostructures directly on the face of optical fibers. Another approach consists of transferring ${ }^{12-14}$ nanostructures fabricated by means of lithography or self-assembly ${ }^{15-17}$ techniques to the fiber facet.

The main disadvantages of the aforementioned fabrication techniques include complexity, time-consuming fabrication steps, expensive and bulky equipment, etc. Moreover, such fabrication methods offer low or poor reproducibility. In addition, current fiber optic plasmonic nanosensors often do not provide any information about the spectroscopic characteristics of the analyte. Instead they monitor the changes in the dielectric constant of the medium that surrounds the plasmonic nanostructure and that can produce a shift in the LSPR peak. 
Here, we report the development of fiber optic nanosensors via plasmon resonance energy transfer (PRET) ${ }^{18-27}$. The latter, is a nanospectroscopy effect that occurs only when the frequencies between the light scattered from AuNPs and those adsorbed by target molecules near the AuNPs match. The PRET technique can be carried out even with a single plasmonic nanoparticle ${ }^{19}$. Current sensors based on PRET are capable of detecting chemical and biological parameters with high sensitivity and specificity. However, they require complex setups that involve dark filed microscopes, hyperspectral cameras, and highly sensitive spectrometers. Conversely, our devices are simpler; they are easy to interrogate, as it will demonstrated in the following paragraphs.

To overcome reproducibility issues, a low cost chemical approach to immobilize functionalized AuNPs onto the end face of a conventional multimode optical fiber (MMF) was developed. Unlike previous works ${ }^{28-31}$ where the immobilization of nanoparticles on the fiber end is an incontrollable process. In our case, the deposition of AuNPs was monitored in real time; this allowed us to know indirectly the relative coverage of the fiber optic surface and to achieve highly reproducible probes.

The sensing capability of our fiber optic nanosensors is demonstrated by detecting specifically copper ions $\left(\mathrm{Cu}^{2+}\right)$ in water. It was found that our nanosensors are 1000 times more sensitive than their counterpart based on microscopy by using the same chemistry previously reported ${ }^{32}$. It is also demonstrated that our devices can reach picomolar detection limits and broad measuring range. Therefore, we believe that the concepts and approaches reported here may lead to the development of a new generation of specific fiber optic plasmonic (bio)chemical nanosensors. 


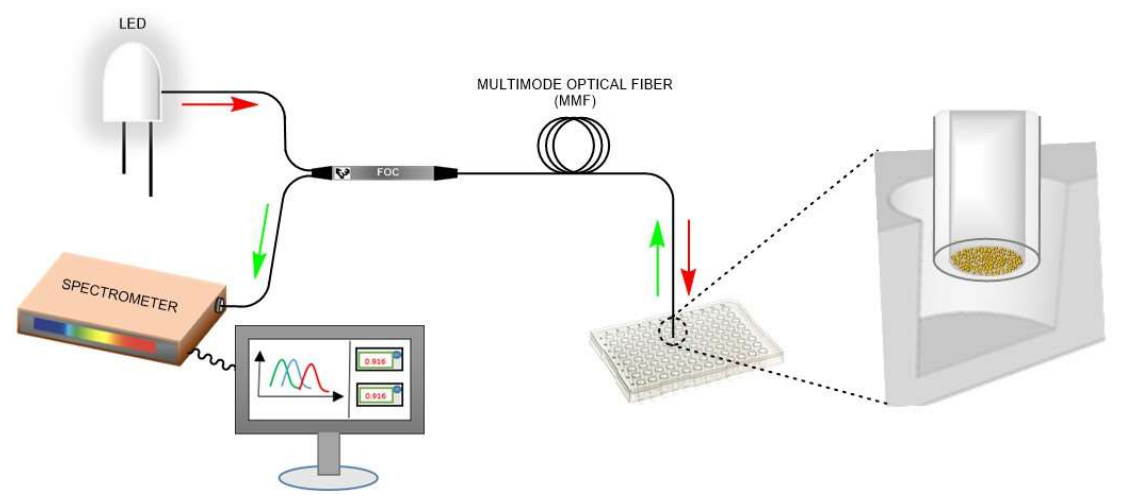

Fig. 1 Schematic of the measuring setup. It contains an LED, a fiber optic coupler (FOC), a spectrometer, multimode optical fiber (MMF), and a small sample container.

\section{Results}

\section{Fabrication and characterization of the nanoprobes}

Figure 1 shows the schematic representation of the setup that was used to monitor in real time the fabrication process of the fiber optic nanoprobes. The same setup was used to characterize the sensors. The setup comprises of a white light LED (MCWHL5, Thorlabs) with emission from 400 to $700 \mathrm{~nm}$, an MMF coupler (TM105R5S1A, Thorlabs), a low-resolution spectrometer (Avantes, mini2048-VI25) connected to a computer and controlled with Avantes software, and a sampling chamber. All the components of our measuring setup are inexpensive and widely available. The cost of all the components to implement our setup was less than 2500 Euros.

Light from the LED was launched to the end face of the MMF by means of the fiber optic coupler. The light reflected from the MMF face passed again through the coupler and reached the spectrometer. In all the experiments, the absorbance spectra were calculated with the following expression: 


$$
A=-\log \left(\frac{S-D}{R-D}\right)
$$

In the above equation, $R$ is the spectrum that is obtained when there are no AuNPs onto the MMF facet, $D$ is the measured spectrum when the LED is turned off, and $S$ is the reflected spectrum from the AuNPs-coated fiber facet. The acquisition of the absorbance spectra was optimized by adjusting the power of the LED or the exposition time of the spectrometer.

The MMF used in the experiments (FG105LCA, Thorlabs) had core/cladding diameter of $105 / 125 \mu \mathrm{m}$. It was cleaved with a common optical fiber cleaver (VF-78, INNO Instrument America). The cleaving process guarantees MMF tips with smooth and flat surfaces. The MMF tips were cleaned in a piranha solution during 30 min, and later, they were modified with 3-aminopropyltriethoxysilane (APTES); an organic linker molecule, to enable covalent attachment of $40 \mathrm{~nm}$-diameter AuNPs. Upon attachment of AuNPs to the fiber facet, we observed an absorption spectrum, indeed the LSPR peak, with maxima centered ca. $540 \mathrm{~nm}$, see Fig. 2a. The intensity of the absorption spectrum increased with time of incubation until it reached a plateau after 45 minutes, see Fig. $2 b$. The peak of the absorption spectra matched the LSPR peak of the $40 \mathrm{~nm}$ AuNPs provided by the manufacturer, thus confirming immobilization of the nanoparticles on the facet of the MMF.

Scanning electron microscopy (SEM) images of the MMF tips showed an increasing density of AuNPs on the fiber facet with time of incubation, a homogeneous distribution of the AuNPs, and a very low number of aggregates, see Fig. $2 \mathrm{c}$ and $2 \mathrm{~d}$. The formation of dimmers and trimmers increased with time of 

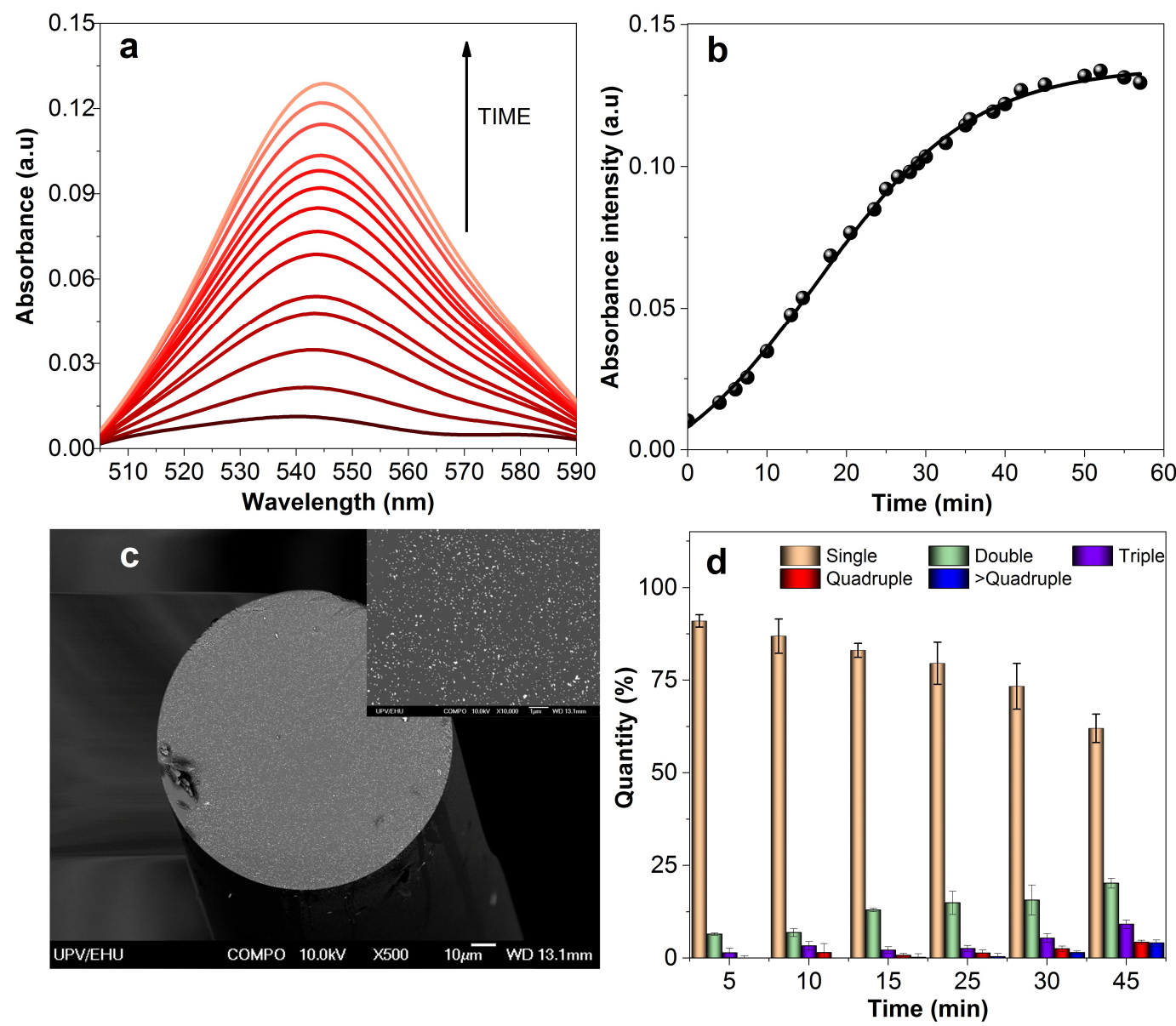

Fig. 2 Study of time deposition. a Evolution of the absorption spectra with incubation time. $\mathbf{b}$ Intensity of the LSPR peak with deposition time. The solid line is a fitting to the data. c SEM image of the MMF facet after 45 minutes of incubation time (Magnification 10,000x. COMPO mode). The inset image is a close-up. $\mathbf{d}$ Percentage of single AuNPs observed at different incubation times. Error bars correspond to the standard errors from 3 samples.

incubation, but single AuNPs remained over $75 \%$ of the total events, see Fig. $2 \mathrm{~d}$. SEM images of MMF facets after different AuNPs immobilization times are shown in Fig. S1.

The measured absorption spectra correlated well with the SEM images. Higher intensities of the LSPR peaks are in good agreement with an increased 
density of AuNPs on the MMF facet. The minute red shift shown in Fig. 2a could be explained by the formation of some aggregates at prolonged incubation times.

The immobilization of AuNPs was reproducible; a coefficient of variation (CV) of $6,2 \%$ in the binding curves between three different samples was observed. An additional advantage of our protocol to immobilize AuNPs is its potential for batch fabrication of nanosensors, something that is not possible with prior nanofabrication techniques.

\section{Nanosensors for selective copper ions detection}

Copper is an essential element in biological processes; however, concentrations higher than $63 \mu \mathrm{M}$ in water may lead to harmful effects for human health ${ }^{33}$. So far, different techniques have been performed to detect $\mathrm{Cu}^{2+}$ in water, as for example, electrochemistry, fluorescence, PRET, colorimetric methods, and surface enhanced Raman spectroscopy ${ }^{34-39}$. However, atomic absorption spectrometry and inductively coupled plasma mass spectroscopy are the gold standard techniques to measure $\mathrm{Cu}^{2+}$ in water. The limit of detection of such expensive techniques is in the micro molar range but they operate in centralized analytical laboratories only. Thus, a stand-alone portable system that enables on-site detection of $\mathrm{Cu}^{2+}$ in water, hence, reducing the time and cost of the analytical process, is highly desirable.

In order to enable $\mathrm{Cu}^{2+}$ detection with our devices, we fabricated nanoprobes where the immobilization of Au nanoparticles was set at 25 minutes to have a good coverage of the MMF facet while avoiding the formation of many aggregates, see Fig. 2b. The MMFs coated with AuNPs were further functionalized with N-[3(trimethoxysilyl)propyl]ethylediamine (TMSen), to form $\mathrm{Cu}^{2+}$ specific ligands on the surface of the AuNPs, see Fig. 3. This functionalization of the AuNPs induced a 
negligible change in the absorption spectra obtained from the MMF facet (see Fig. S2).

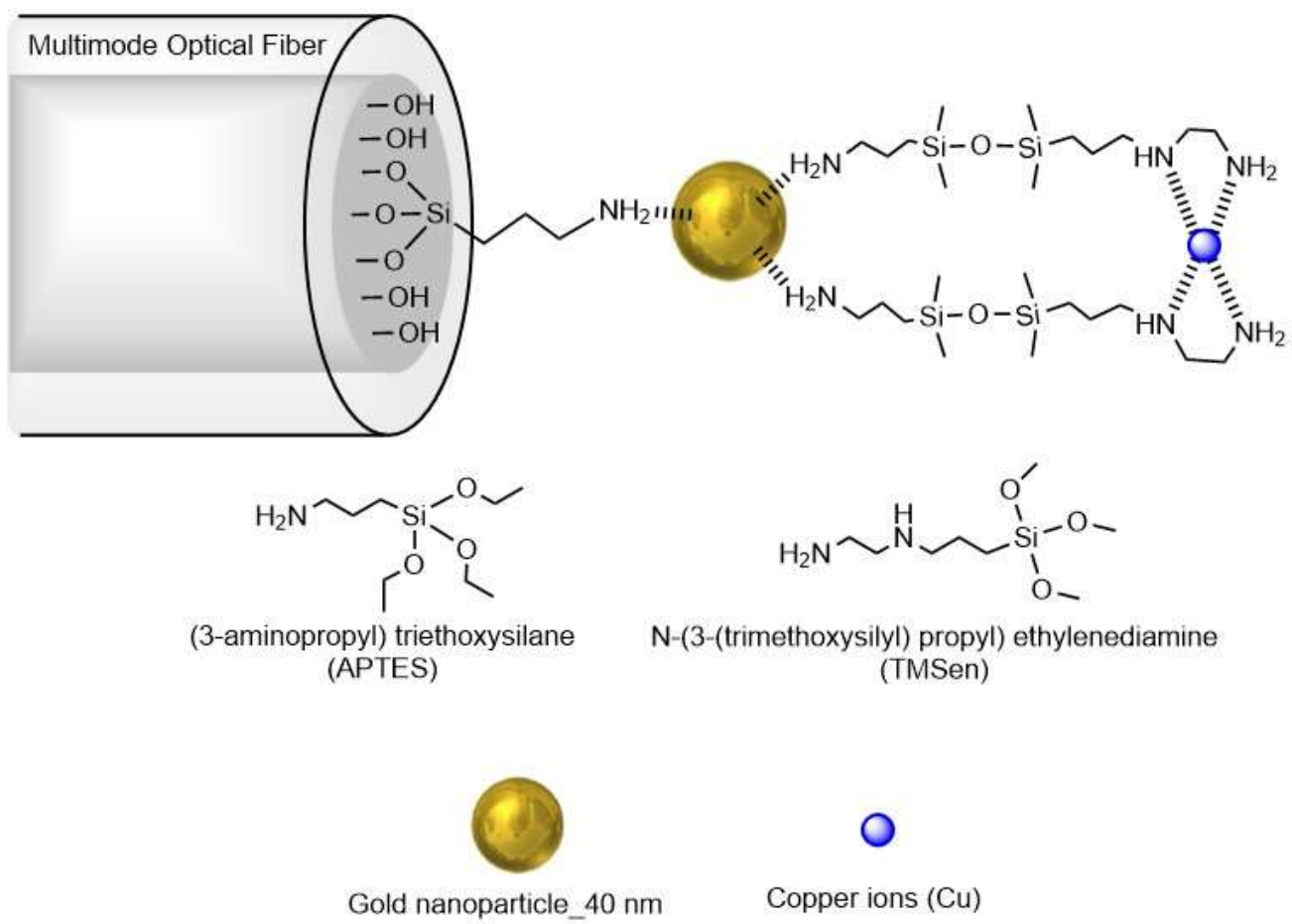

Fig. 3 PRET surface. Schematic representation of the facet of an MMF with AuNPs attached and functionalized with TMSen.

To evaluate the sensing performance of the fiber optic nanoprobes, they were immersed in a series of aqueous solutions containing increasing concentrations of $\mathrm{Cu}^{2+}$ ions ranging from $10^{-12}$ to $10^{-3} \mathrm{M}$. The results of our experiments are summarized in Fig. 4. From Fig. 4a, it can be observed that the intensity of the LSPR peak decreases as the concentration of $\mathrm{Cu}^{2+}$ in the solution increases. The measuring range of our fiber optic nanosensor was found to be linear; from $10^{-12}$ up to $10^{-4} \mathrm{M}$ with saturation occurring from $10^{-3} \mathrm{M}$ onwards, see the calibration curve shown in Fig. $4 b$. 
In such a figure, the plotted data were calculated by the expression: $100 *\left(1-I / I_{0}\right)$, where $I$ corresponds to the maximum signal intensity of each concentration and $I_{0}$ to $0 \mu \mathrm{M}$ concentration, this means, no $\mathrm{Cu}^{2+}$.

Efficient complexation of $\mathrm{Cu}^{2+}$ by the nanoprobes was further confirmed by XPS (Fig. S3). Additionally, a competitive binding assay was performed using ethylenediaminetetraacetic acid (EDTA). EDTA is a chelating agent with stronger affinity for $\mathrm{Cu}^{2+}$ ions than TMSen complex. Incubation of $\mathrm{Cu}^{2+}$ saturated PRET surfaces in a $0.1 \mathrm{M}$ solution of EDTA, resulted in the recovery of the LSPR peak intensity of the MMF-AuNPs-TMSen obtained prior to incubation with $\mathrm{Cu}^{2+}$ ions. This observation was in agreement with the removal of $\mathrm{Cu}^{2+}$ ions from the PRET surfaces by EDTA solutions (Fig. S4).

The experiments described above were repeated with AuNPs-coated MMF facets that were not modified with TMSen. The results of such experiments are shown in Fig. 4b. No significant changes in LSPR peak were observed thus confirming that $\mathrm{Cu}^{2+}$ ions did not interact with the AuNPs in the absence of amine functional groups.

Selectivity tests were carried out for the $\mathrm{Cu}^{2+}$ fiber optic PRET nanosensors. They did not show a significant response in the absorption spectra in the presence of different heavy metal ions $\left(\mathrm{Co}^{2+}, \mathrm{Pb}^{2+}, \mathrm{Ni}^{2+}\right)$ and other ions $\left(\mathrm{Na}^{+}\right)$that usually accompany $\mathrm{Cu}^{2+}$ ions in water, see Fig. 5a. This agrees with the fact that the AuNPs were modified with $\mathrm{Cu}^{2+}$ specific ligands, thus, other metal ions could not be bound to the surface of AuNPs. However, all the ions produced an unspecific LSPR shift (see Fig. 5b). The reason for the LSPR shift in the presence of $\mathrm{Cu}^{2+}$ ions and other metals in the solution is related to changes of the dielectric constant, while the decrease in the LSPR peak intensity is related to the PRET effect. 

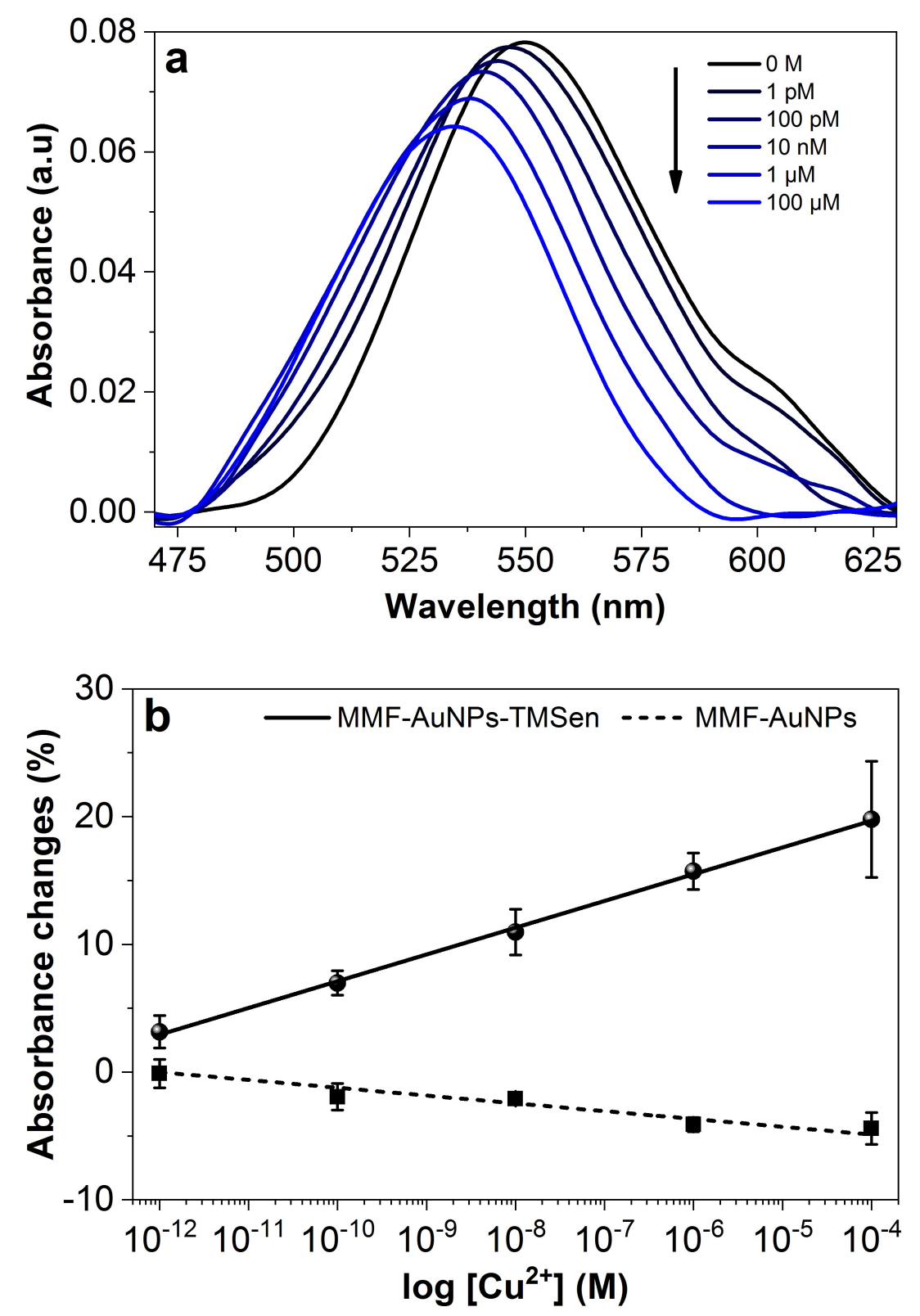

Fig. 4 Nanosensor response upon complexation of $\mathrm{Cu}^{2+}$. a Absorption spectra of observed in different aqueous solutions with increasing concentrations of $\mathrm{Cu}^{2+}$ ions. $\mathbf{b}$ Corresponding calibration plot. The solid line is a fitting to the experimental data. $\mathrm{Cu}^{2+}$ ions detection using no-functionalized nanoprobes are shown with the data fitted with the dashed line. 

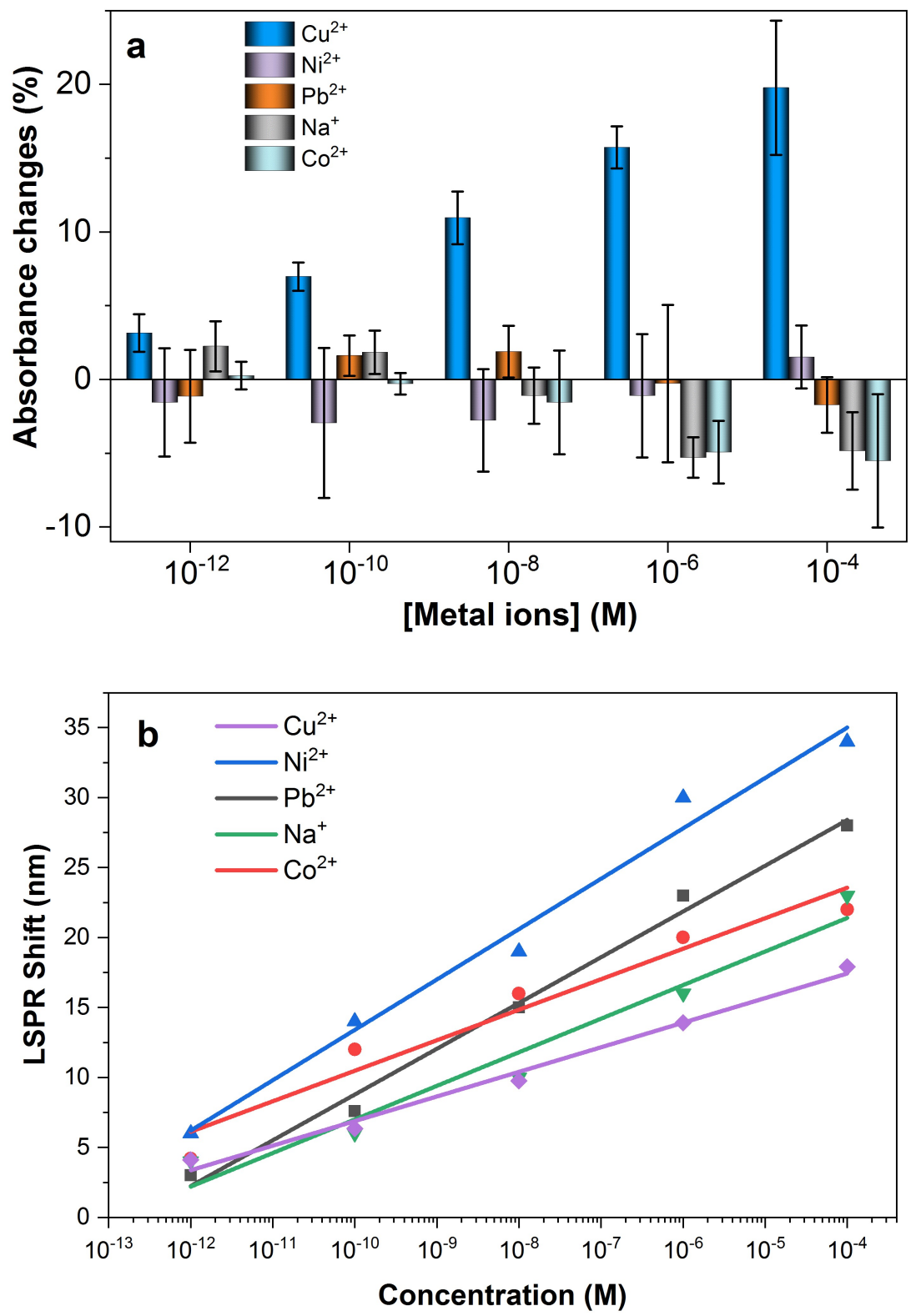

Fig. 5 Interference studies. a Bars diagram showing the effect of $\mathrm{Cu}^{2+}, \mathrm{Ni}^{2+}$, $\mathrm{Pb}^{2+}, \mathrm{Na}^{2+}$, and $\mathrm{Co}^{2+}$ on the intensity of the LSPR peak. Error bars correspond to the standard errors from 3 tested samples. b LSPR shifts observed with the presence of same ions in a at concentrations ranging from $10^{-12}$ to $10^{-4} \mathrm{M}$. In all cases, the incubation time was 5 minutes.

\section{Discussion}

The light scattered from $40 \mathrm{~nm}$-diameter AuNPs has an LSPR peak close to $540 \mathrm{~nm}$. Therefore, the absorbance spectrum of an MMF coated with such nanoparticles was 
expected to exhibit a peak ca. $540 \mathrm{~nm}$; see Eq. (1) for the definition of absorbance used in this work. The results shown in Fig. 4a confirm that the observed spectra corresponded to the LSPR peaks of the $40 \mathrm{~nm}$-diameter AuNPs. On the other hand, ethylenediamine copper complex has optical absorption in the visible range with a peak centered at $569 \mathrm{~nm}$. Therefore, there is an overlapping between the LSPR spectral peak of the metallic nanoparticles and the absorption peaks of the $\mathrm{Cu}^{2+}$ complex formed on top of the nanoparticles. This is what enables energy transfer between the AuNPs and the coordinated $\mathrm{Cu}^{2+}$ ions ${ }^{32}$.

When the AuNPs deposited on the MMF facet are illuminated with white light, the free electrons of the nanoparticles enter in resonance. The energy stored in such resonant electrons can be transferred to $\mathrm{Cu}^{2+}$ ions located close to the nanoparticles. Consequently, the intensity of the absorbance spectra of the fiber nanoprobe changes. It is worth noting that the absorbance changes in proportion to the concentration of $\mathrm{Cu}^{2+}$ ions, see Fig. 4a. The results shown in Fig. 4 suggest that as the concentration of copper ions increases more energy is transferred from the nanoparticles to the ions.

Our observations are in agreement with previous reports that showed a decrease in the intensity of the Rayleigh scattering of single nanoparticles functionalized with $\mathrm{Cu}^{2+}$ chelating agents upon $\mathrm{Cu}^{2+}$ complexation ${ }^{32}$. A similar response could be expected from other transition metal ions such as $\mathrm{Co}^{2+}$ and $\mathrm{Ni}^{2+}$ that also form complexes, which have absorption bands overlapping the Rayleigh scattering of the nanoparticles, but it was not obtained. The large specificity for $\mathrm{Cu}^{2+}$ must be related to its higher affinity for ethylenediamine compared to $\mathrm{Co}^{2+}$ and $\mathrm{Ni}^{2+}$ as well as an intense absorption by the $\mathrm{Cu}^{2+}$ complex in the visible range ${ }^{40}$ (see supporting information) . 
It can be observed from Fig. 4a that the absorbance peak shifts to the blue as the concentration of $\mathrm{Cu}^{2+}$ increases. For our measurements such a shift was not relevant as it was unspecific for several metal ions. Instead, the height or intensity of the peak was specifically correlated with the concentration of $\mathrm{Cu}^{2+}$ in relation with the chemistry used to modify the nanoprobes.

In comparison with other optical sensors for the detection of $\mathrm{Cu}^{2+}$, the PRETbased fiber optic platform reported here showed 1000 times higher sensitivity than its counterpart based on dark field microscopy, and a larger analyzable range of concentrations. The lower concentration of $\mathrm{Cu}^{2+}$ measured was $1 \mathrm{pM}$, and the theoretical LOD calculated as 3 times de signal to noise ratio, was $0.2 \mathrm{pM}$. The performance of our device and that of other optical techniques are summarized in Table S1.

\section{Conclusions}

In summary, we have reported on the development of PRET-based fiber optic nanosensors that may be incorporated in inexpensive, portable detection systems that could be hold on the palm of a hand. The nanosensors proposed here are much simpler to fabricate than other LSPR sensors that require sophisticated nanofabrication techniques. In our approach, the sensor fabrication process can be monitored in real time; hence, it can be controlled.

The potential of our nanosensors for the detection of $\mathrm{Cu}^{2+}$ ions in water was demonstrated. Our nanosensors are 1,000 more sensitivity than previous reported PRET sensors. The significant difference in sensitivity lies on the efficient lightAuNPs interaction as it takes place on the facet of a conventional multimode optical 
fiber. As compared with current equipment for $\mathrm{Cu}^{2+}$ ions detection, our sensing approach can be hundred times cheaper.

In contrast to previous PRET sensors that use a dark field microscope to record the scattering spectra of AuNPs immobilized on a glass surface, the interrogation of our nanosensors is quite simple. It involves low cost, small size, and widely available components such as a white light LED, an optical fiber coupler, and a miniature spectrometer.

Our fiber optic nanosensors provide high specificity through the PRET phenomena and ultra-high sensitivity thanks to its LSPR nature that is further leveraged by depositing the antenna surfaces directly on the face of an optical fiber.

The multiplexing of our devices is feasible with optical fiber switches, which are widely used in optical fiber technology. The incorporation of a switch in the setup shown above (Fig. 1) is straightforward. Therefore, differently functionalized nanosensors would specifically detect a number of different analytes.

We believe that the sensing concept presented here will be a breakthrough as it will enable the development of ultrasensitive and specific fiber optic plasmonic (bio)chemical nanosensors. Such sensors will find application in many different high impact fields such as on-site monitoring of heavy metals in water or even ultrasensitive detection of protein, nucleic acids, and many other analytes.

To the best of our knowledge, this is the first report of the combination of LSPR and the specific chemical fingerprint by PRET on the face of MMFs. It opens up the possibility of generating a new and powerful detection method for a wide number of areas.

\section{Materials and methods}




\section{Chemicals}

All chemicals were purchased from Sigma-Aldrich and used as received from the supplier without further purification. All water used was Mili-Q ultrapure grade (18.2 $\mathrm{M} \Omega \mathrm{cm}) .40 \mathrm{~nm}$ diameter citrated capped spherical gold nanoparticles aqueous solutions (particle concentration $=6.2 \times 10^{-10}$ particles $\cdot \mathrm{mL}^{-1}$ ) were purchased from Nanovex Biotechnologies S.L. (Spain).

\section{Preparation of PRET surfaces}

The end-face of the MMF was cleaved by using an optical fiber cleaver (VF-78, INNO Instrument America). The cut angle was verified by optical microscopy, being perpendicular to the cross section of the incident light. MMFs were cleaned in a piranha solution during 30 min and rinsed with DI water. Later, MMFs were modified with 3-aminoproyltriethoxysilane (APTES) by incubation in $1 \mathrm{mM}$ APTES solution in isopropanol for 90 minutes. MMFs were then rinsed out with a solution of isopropanol-water (1:1). Later, AuNPs were immobilized on the APTES-modified MMFs by immersion of the fibers in a $6.2 \times 10^{-10}$ particles/mL AuNPs aqueous suspension during $25 \mathrm{~min}$. Subsequently, freshly prepared MMF-AuNPs were immersed in 1mM APTES solution in isopropanol for $90 \mathrm{~min}$. The silanefunctionalized MMF-AuNPs were washed with a solution of isopropanol-water (1:1) and were immersed in a $0,1 \mathrm{M} \mathrm{HCl}$ solution for hydrolysis of the methoxy groups of APTES (1 h), and rinsed with water. Finally, the gold plasmonic probes were immersed into $1 \mathrm{mM} \mathrm{N}$-[3-(trimethoxysilyl)propyl]ethylediamine (TMSen) solution for 2 $\mathrm{h}$ to obtain the MMF-AuNPs-TMSen, or nanoprobes, and then it was rinsed with Mili$\mathrm{Q}$ water for $5 \mathrm{~min}$. MMF-PRET platforms were stocked in Mili-Q water and protected from light. 


\section{Scanning Electronic Miscroscopy (SEM) characterization}

The presence of AuNPs attached to the end-face of the MMFs was confirmed by SEM. Images were acquired in a SEM of type JEOL JSM-7000-F (JEOL, Japan) at $10 \mathrm{kV}$ acceleration voltage. MMFs were placed in metallic holder aligned vertically. A chromium monolayer of $5 \mathrm{~nm}$ was deposited by sputtering for surface metallization. The aggregation ratio was obtained by the public domain software ImageJ $1,51 \mathrm{~d}$ (National Institutes of Health, USA).

\section{PRET sensing}

Different MMF-AuNPs-TMSen were immersed into aqueous solutions of varying concentrations of metal ions in a final volume of $300 \mu \mathrm{L}$, using black flat-well (NUNC 96 wells) plates. All components were used at the same range of concentrations (from $10^{-12}$ to $10^{-4} \mathrm{M}$ ). The ions solutions were prepared from their following salts: $\mathrm{CuCl}_{2}, \mathrm{~Pb}\left(\mathrm{NO}_{3}\right)_{2}, \mathrm{Co}\left(\mathrm{NO}_{3}\right)_{2}, \mathrm{Ni}\left(\mathrm{NO}_{3}\right)_{2}$ and $\mathrm{NaCl}$. In all the measurements, the spectra of the resulting mixtures were recorded after $5 \mathrm{~min}$.

\section{Data availability}

The authors declare that the data supporting the findings of this study are available within the paper and its supplementary information files.

Supporting Information Available: The following files are available free of charge: Supporting Information. Brief description of contents: SEM images of the facets of optical fibers with different concentrations of AuNPs. Spectra from functionalized optical fibers. XPS spectra of functionalized glass surfaces. UV-vis Spectra of TMSem complexed with $\mathrm{Cu}^{2+}, \mathrm{Co}^{2+}$ and $\mathrm{Ni}^{2+}$. Spectra of MMF-PRET-Cu${ }^{2+}$ before and after incubation with $0.1 \mathrm{M}$ EDTA. 


\section{Acknowledgements}

LBD, FBL, JB, ACS acknowledge funding support from Gobierno de España, Ministerio de Economia y Competitividad, with Grant No. BIO2016-80417-P and European Union funds: DNASURF (H2020-MSCA-RISE-778001). They also acknowledge funding support from Departamento de Educación del Gobierno Vasco Grant No. IT1271-19. JV, JZ, and AO acknowledge funding support from the Fondo Europeo de Desarrollo Regional (FEDER) and the Ministerio de Economia y Competitividad (Spain) under projects PGC2018-101997-B-I00 and RTI2018094669-B-C31, and also from the Departamento de Educación del Gobierno Vasco Grant No. IT933-16. The authors thank for technical and human support provided by electronic microscopy and material microanalysis services from Advanced Research Facilities (SGIker) of the University of the Basque Country UPV/EHU.

\section{Author Contributions}

$\mathrm{JB}$ and $\mathrm{AOG}$ designed the work, acquired data, interpreted results, and drafted the manuscript. ACS acquired and interpreted data. LBD, JV, FBL, and JZ conceived and designed the work, interpreted the results. JV and LBD wrote the final version of the manuscript with the approval of all the authors.

\section{Conflict of interest}

The authors declare no conflict of interest 


\section{References}

1. Sepúlveda, B.; Angelomé, P. C.; Lechuga, L. M.; Liz-Marzán, L. M., LSPR-based nanobiosensors. Nano Today 2009, 4, 244-251, DOI:

10.1016/j.nantod.2009.04.001

2. Mayer, K. M.; Hafner, J. H., Localized Surface Plasmon Resonance Sensors. Chemical Reviews 2011, 111, 3828-3857, DOI: 10.1021/cr100313v

3. Huang, X.; El-Sayed, I. H.; Qian, W.; El-Sayed, M. A., Cancer Cell Imaging and Photothermal Therapy in the Near-Infrared Region by Using Gold Nanorods. Journal of the American Chemical Society 2006, 128, 2115-2120, DOI: 10.1021/ja057254a

4. Aćimović, S. S.; Ortega, M. A.; Sanz, V.; Berthelot, J.; Garcia-Cordero, J. L.; Renger, J.; Maerkl, S. J.; Kreuzer, M. P.; Quidant, R., LSPR Chip for Parallel, Rapid, and Sensitive Detection of Cancer Markers in Serum. Nano Lett. 2014, 14, 2636-2641, DOI: 10.1021/nl500574n

5. Caucheteur, C.; Guo, T.; Albert, J., Review of plasmonic fiber optic biochemical sensors: improving the limit of detection. Analytical and Bioanalytical Chemistry 2015, 407, 3883-3897, DOI: 10.1007/s00216-014-8411-6

6. Sanders, M.; Lin, Y.; Wei, J.; Bono, T.; Lindquist, R. G., An enhanced LSPR fiber-optic nanoprobe for ultrasensitive detection of protein biomarkers.

Biosensors and Bioelectronics 2014, 61, 95-101, DOI: 10.1016/j.bios.2014.05.009

7. Lin, Y.; Zou, Y.; Lindquist, R. G., A reflection-based localized surface plasmon resonance fiber-optic probe for biochemical sensing. Biomed. Opt. Express 2011, 2, 478-484, DOI: 10.1364/BOE.2.000478

8. Lin, Y. B.; Zou, Y.; Mo, Y. Y.; Guo, J. P.; Lindquist, R. G., E-Beam Patterned Gold Nanodot Arrays on Optical Fiber Tips for Localized Surface Plasmon Resonance Biochemical Sensing. Sensors 2010, 10, 9397-9406, DOI: 10.3390/s101009397

9. Kim, H.-M.; Uh, M.; Jeong, D. H.; Lee, H.-Y.; Park, J.-H.; Lee, S.-K., Localized surface plasmon resonance biosensor using nanopatterned gold particles on the surface of an optical fiber. Sensors and Actuators B: Chemical 2019, 280, 183191, DOI: 10.1016/j.snb.2018.10.059

10.Consales, M.; Ricciardi, A.; Crescitelli, A.; Esposito, E.; Cutolo, A.; Cusano, A., Lab-on-Fiber Technology: Toward Multifunctional Optical Nanoprobes. ACS Nano 2012, 6, 3163-3170, DOI: 10.1021/nn204953e

11. Smythe, E. J.; Cubukcu, E.; Capasso, F., Optical properties of surface plasmon resonances of coupled metallic nanorods. Opt. Express 2007, 15, 7439-7447, DOI: 10.1364/oe.15.007439

12.Jia, P.; Yang, Z.; Yang, J.; Ebendorff-Heidepriem, H., Quasiperiodic Nanohole Arrays on Optical Fibers as Plasmonic Sensors: Fabrication and Sensitivity Determination. ACS Sensors 2016, 1, 1078-1083, DOI: 10.1021/acssensors.6b00436

13.Siegfried, T.; Ekinci, Y.; Martin, O. J. F.; Sigg, H., Engineering Metal Adhesion Layers That Do Not Deteriorate Plasmon Resonances. ACS Nano 2013, 7, 2751 2757, DOI: $10.1021 / \mathrm{nn} 4002006$

14.Jia, P.; Yang, J., A plasmonic optical fiber patterned by template transfer as a highperformance flexible nanoprobe for real-time biosensing. Nanoscale 2014, 6, 8836-8843, DOI: 10.1039/C4NR01411A

15.Polley, N.; Basak, S.; Hass, R.; Pacholski, C., Fiber optic plasmonic sensors: Providing sensitive biosensor platforms with minimal lab equipment. Biosensors and Bioelectronics 2019, 132, 368-374, DOI: 10.1016/j.bios.2019.03.020 
16.Pisco, M.; Galeotti, F.; Quero, G.; Grisci, G.; Micco, A.; Mercaldo, L. V.; Veneri, P. D.; Cutolo, A.; Cusano, A., Nanosphere lithography for optical fiber tip nanoprobes. Light: Science \& Applications 2017, 6, e16229-e16229, DOI: 10.1038/Isa.2016.229

17.Liang, Y.; Yu, Z.; Li, L.; Xu, T., A self-assembled plasmonic optical fiber nanoprobe for label-free biosensing. Sci Rep 2019, 9, 7379, DOI: 10.1038/s41598-019-43781-8

18.Cao, Y.; Xie, T.; Qian, R. C.; Long, Y. T., Plasmon Resonance Energy Transfer: Coupling between Chromophore Molecules and Metallic Nanoparticles. Small 2017, 13, DOI: 10.1002/smll.201601955

19.Li, S. S.; Kong, Q. Y.; Zhang, M.; Yang, F.; Kang, B.; Xu, J. J.; Chen, H. Y., Plasmon-Resonance-Energy-Transfer-Based Spectroscopy on Single Nanoparticles: Biomolecular Recognition and Enzyme Kinetics. Anal. Chem. 2018, 90, 3833-3841, DOI: 10.1021/acs.analchem.7b004467

20.Kim, Y.; Park, J. Y.; Kim, H. Y.; Lee, M.; Yi, J.; Choi, I., A single nanoparticlebased sensor for hydrogen peroxide ( $\mathrm{H} 2 \mathrm{O} 2$ ) via cytochrome c-mediated plasmon resonance energy transfer. Chem. Commun. 2015, 51, 15370-15373, DOI: $10.1039 / \mathrm{c5cc05327g}$

21.Shi, L.; Jing, C.; Gu, Z.; Long, Y. T., Brightening Gold Nanoparticles: New Sensing Approach Based on Plasmon Resonance Energy Transfer. Sci Rep 2015, 5, 7, DOI: 10.1038/srep10142

22.Qu, W. G.; Deng, B.; Zhong, S. L.; Shi, H. Y.; Wang, S. S.; Xu, A. W., Plasmonic resonance energy transfer-based nanospectroscopy for sensitive and selective detection of 2,4,6-trinitrotoluene (TNT). Chem. Commun. 2011, 47, 1237-1239, DOI: $10.1039 / \mathrm{c0cc02752a}$

23.Liu, G. L.; Long, Y. T.; Choi, Y.; Kang, T.; Lee, L. P., Quantized plasmon quenching dips nanospectroscopy via plasmon resonance energy transfer. Nat. Methods 2007, 4, 1015-1017, DOI: 10.1038/nmeth1133

24.Choi, Y. H.; Kang, T.; Lee, L. P., Plasmon Resonance Energy Transfer (PRET)based Molecular Imaging of Cytochrome c in Living Cells. Nano Lett. 2009, 9, 8590, DOI: $10.1021 / \mathrm{nl} 802511 \mathrm{z}$

25.Xie, T.; Li, M.; Long, Y.-T., Dual-channel signals for intracellular mRNA detection via a PRET nanosensor. Chem. Commun. 2017, 53, 7768-7771, DOI: 10.1039/C7CC02864D

26.Gao, M. X.; Zou, H. Y.; Li, Y. F.; Huang, C. Z., General Sensitive Detecting Strategy of lons through Plasmonic Resonance Energy Transfer from Gold Nanoparticles to Rhodamine Spirolactam. Anal. Chem. 2017, 89, 1808-1814, DOI: 10.1021/acs.analchem.6b04124

27.Yan, X.; Xia, C.; Chen, B.; Li, Y. F.; Gao, P. F.; Huang, C. Z., Enzyme Activity Triggered Blocking of Plasmon Resonance Energy Transfer for Highly Selective Detection of Acid Phosphatase. Anal. Chem. 2020, 92, 2130-2135, DOI: 10.1021/acs.analchem.9b04685

28. Mitsui, K.; Handa, Y.; Kajikawa, K. J. A. P. L., Optical fiber affinity biosensor based on localized surface plasmon resonance. 2004, 85, 4231-4233, DOI: 10.1063/1.1812583

29.Sciacca, B.; Monro, T. M., Dip Biosensor Based on Localized Surface Plasmon Resonance at the Tip of an Optical Fiber. Langmuir 2014, 30, 946-954, DOI: 10.1021/la403667q

30.Kim, H.-M.; Park, J.-H.; Jeong, D. H.; Lee, H.-Y.; Lee, S.-K., Real-time detection of prostate-specific antigens using a highly reliable fiber-optic localized surface 
plasmon resonance sensor combined with micro fluidic channel. Sensors and Actuators B: Chemical 2018, 273, 891-898, DOI: 10.1016/j.snb.2018.07.007

31.Camara, A. R.; Gouvêa, P. M. P.; Dias, A. C. M. S.; Braga, A. M. B.; Dutra, R. F.; de Araujo, R. E.; Carvalho, I. C. S., Dengue immunoassay with an LSPR fiber optic sensor. Opt. Express 2013, 21, 27023-27031, DOI: 10.1364/OE.21.027023

32.Choi, Y.; Park, Y.; Kang, T.; Lee, L. P., Selective and sensitive detection of metal ions by plasmonic resonance energy transfer-based nanospectroscopy. Nat. Nanotechnol. 2009, 4, 742-746, DOI: 10.1038/nnano.2009.258

33.Georgopoulos, P. G.; Roy, A.; Yonone-Lioy, M. J.; Opiekun, R. E.; Lioy, P. J., Environmental copper: Its dynamics and human exposure issues. J. Toxicol. Env. Health-Pt b-Crit. Rev. 2001, 4, 341-394, DOI: 10.1080/109374001753146207

34.Frag, E. Y.; Abdel Hameed, R. M., Preparation, characterization and electrochemical application of $\mathrm{CuNiO}$ nanoparticles supported on graphite for potentiometric determination of copper ions in spiked water samples. Microchemical Journal 2019, 144, 110-116, DOI: 10.1016/j.microc.2018.08.060

35.Han, J.; Tang, X.; Wang, Y.; Liu, R.; Wang, L.; Ni, L., A quinoline-based fluorescence "on-off-on" probe for relay identification of $\mathrm{Cu} 2+$ and $\mathrm{Cd} 2+$ ions. Spectrochimica Acta Part A: Molecular and Biomolecular Spectroscopy 2018, 205, 597-602, DOI: 10.1016/j.saa.2018.07.081

36.Jing, C.; Shi, L.; Liu, X. Y.; Long, Y. T., A single gold nanorod as a plasmon resonance energy transfer based nanosensor for high-sensitivity $\mathrm{Cu}(\mathrm{II})$ detection. Analyst 2014, 139, 6435-6439, DOI: 10.1039/c4an01456a

37.Liu, S.; Li, X., Colorimetric detection of copper ions using gold nanorods in aquatic environment. Materials Science and Engineering: B 2019, 240, 49-54, DOI: 10.1016/j.mseb.2019.01.008

38.Liu, Y.; Wu, Y.; Guo, X.; Wen, Y.; Yang, H., Rapid and selective detection of trace Cu2+ by accumulation- reaction-based Raman spectroscopy. Sensors and Actuators B: Chemical 2019, 283, 278-283, DOI: 10.1016/j.snb.2018.12.043

39.Ngamdee, K.; Chaiendoo, K.; Saiyasombat, C.; Busayaporn, W.; Ittisanronnachai, S.; Promarak, V.; Ngeontae, W., Highly selective circular dichroism sensor based on d-penicillamine/cysteaminecadmium sulfide quantum dots for copper (II) ion detection. Spectrochimica Acta Part A: Molecular and Biomolecular Spectroscopy 2019, 211, 313-321, DOI: 10.1016/j.saa.2018.12.027

40.Smith, R. M.; Martell, A. E., Critical stability constants: inorganic complexes. Springer: 1976; Vol. 4. 
For Table of Contens only

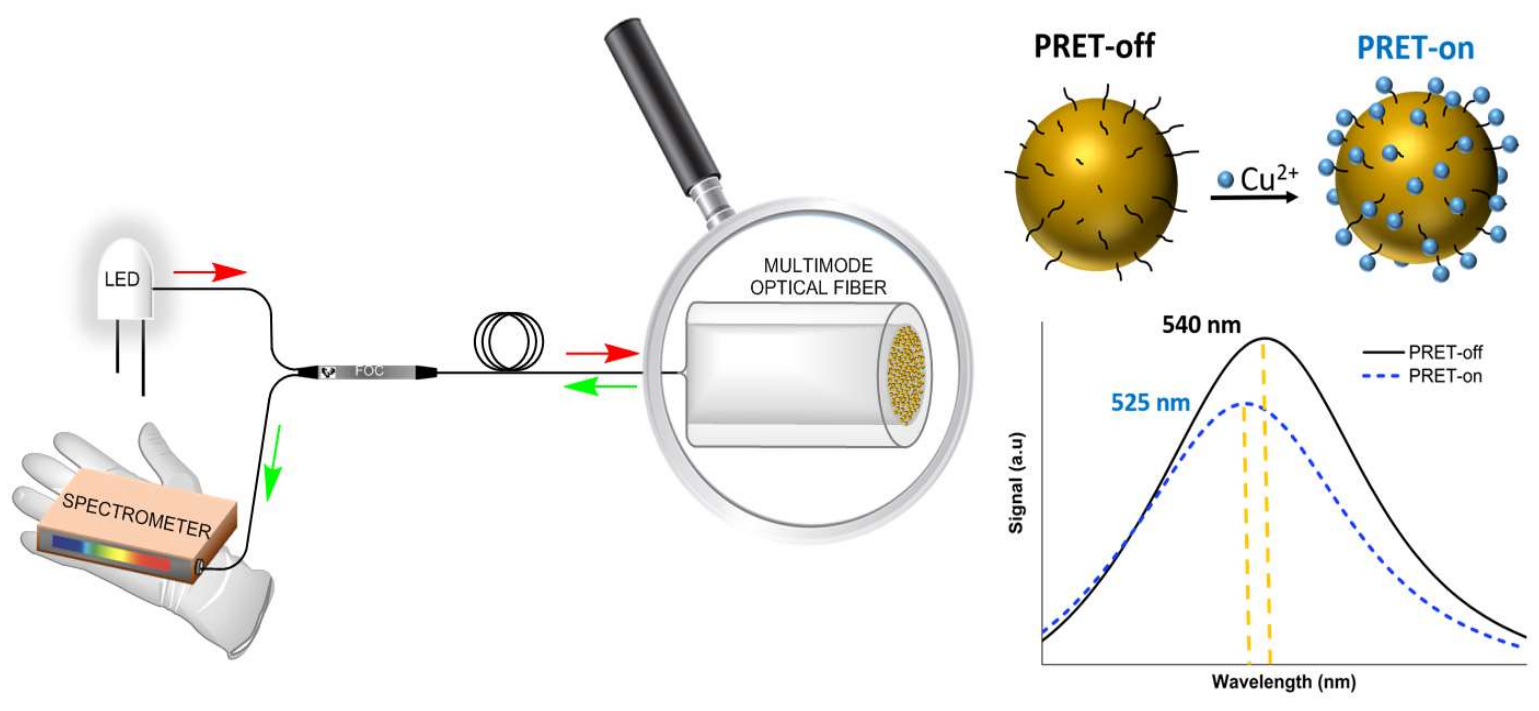

